\title{
Das Nachhaltigkeitskonzept als rechtliche Kategorie im Spannungs- feld zwischen staatlichen Regulierungsinteressen und Investorschutz
}

\section{Katja Gehne}

Das globale Netz des internationalen Investitionsrechts hat sich in den letzten Jahren stark ausgedehnt. Investitionsschutzregelungen finden sich nicht nur in über 2500 bilateralen Investitionsabkommen, sondern auch in regionalen Freihandelsabkommen oder Präferenzabkommen mit Entwicklungsländern verankert. Schutzregeln, die, wie z.B. vor Enteignung oder unfairer Behandlung, nicht allein ein kommerzielles Verhältnis betreffen, sondern Einfluss auf die Regulierungsfreiheit der Staaten nehmen, können zu Interessenskonflikten zwischen dem öffentlichen Interesse an sozial oder ökologisch motivierten Bestimmungen einerseits und Investitionsschutzinteressen andererseits führen. Das internationale Investitionsrecht sieht sich heute mit der Frage konfrontiert, wie dem Spannungsverhältnis der unterschiedlichen Interessenssphären im Anwendungsbereich von internationalen Investitionsschutzbestimmungen und anderen internationalen Standards begegnet werden kann. Der Artikel zeigt auf, dass das Konzept der Nachhaltigen Entwicklung, wie es sich im UN-Kontext in den letzten 20 Jahren herausgebildet hat, hier nützliche Anhaltspunkte liefern kann. Mit dem Vertrag von Lissabon hat die EU Kompetenzen im Bereich der internationalen Investitionspolitik erworben. Sie ist im Verhältnis zu Entwicklungsländern, aber auch gemäß ihrer politischen Leitlinien, an das Leitbild der Nachhaltigen Entwicklung gebunden. Sie kann hier einen wichtigen Beitrag zu einer kohärenten Rechtsentwicklung leisten.

Der Beitrag wird in dem Sammelband Bungenberg/Griebel/Hindelang (Hrsg.), Internationales Investitionschutzrecht und Europarecht, 2010, erscheinen.

NCCR TRADE WORKING PAPERS are preliminary documents posted on the NCCR Trade Regulation website (<www.nccr-trade.org>) and widely circulated to stimulate discussion and critical comment. These papers have not been formally edited. Citations should refer to a "NCCR Trade Working Paper", with appropriate reference made to the author(s). 


\section{Einleitung}

Mit dem folgenden Beitrag soll das Augenmerk auf das Konzept der Nachhaltigen Entwicklung gelenkt werden, wenn es darum geht, das Spannungsverhältnis zwischen Investorschutz und staatlichen Regulierungsinteressen im Gemeinwohlinteresse in rechtliche Kategorien zu fassen. Zwar besteht bisher keine gängige Praxis, das Nachhaltigkeitskonzept in internationale Investitionsabkommen (IIA) aufzunehmen oder es als rechtliche Leitlinie in der internationalen Schiedsgerichtsrechtsprechung heranzuziehen. Das Konzept ist bisher auch nicht vollumfänglich als völkerrechtliches Rechtsprinzip anerkannt ${ }^{1}$. Dennoch ist es für den Zusammenhang des internationalen Investitionsrechts nicht ohne Bedeutung. Zum einen hat das Konzept der Nachhaltigen Entwicklung, seit es in den 1990er Jahren zur UN-Entwicklungsstrategie avancierte, Eingang in verschiedene internationale Vertragswerke gefunden, die für das internationale Investitionsrecht relevant werden können. So ist es z.B. in der Präambel des WTO Marrakesh Abkommens verankert ${ }^{2}$, was dazu führte, dass der WTO Appellate Body hieraus weit reichende Interpretationsstandards ableitete, die mit Blick auf die frühere GATT-Rechtsprechung ein neues Paradigma im Umgang mit anderen, überlappenden umwelt- und gesellschaftsrelevanten Rechtsbereichen einläutete ${ }^{3}$. Zum anderen hat der Internationale Gerichtshof (IGH) das Konzept anlässlich eines ungarischslowakischen Staudammprojekts (Gabcikovo-Nagymaros-Fall) als völkerrechtliche Kategorie (Rechtskonzept) mit spezifischen Rechtsinhalten herangezogen; mit erheblichen Folgen für den Ausgang der Entscheidung.

Mit den neuen Kompetenzregelungen der EU für ausländische Direktinvestitionen im Vertrag von Lissabon (Art. 206, 207 AEUV), werden sich künftig im investitionsrechtlichen Kontext auch Fragen im Hinblick auf das Nachhaltigkeitskonzept stellen. Nicht nur hat das Nachhaltigkeitskonzept im Rahmen der EU Vertragswerke seit dem Vertrag von Maastricht erhebliche Bedeutung erlangt ${ }^{4}$, auch wenn sich dies anders als im WTO-Kontext bis heute nicht explizit in der Rechtsprechung des EuGH widerspiegelt ${ }^{5}$. Mit Blick auf die ge-

\footnotetext{
${ }^{1}$ Bernhard Braune, Rechtsfragen der nachhaltigen Entwicklung im Völkerrecht, 2005.

2 Vgl. Abs. 1 der Präambel des WTO-Abkommens (Marrakesh-Abkommen) vom 15. April 1994.

${ }^{3}$ WTO-Appellate Body, US-Shrimp, WT/DS58/AB/R, 12. Oktober 1998, Rn. 129 ff.; vgl. zur Bedeutung des Nachhaltigkeitskonzept im internationalen Recht, Nico Schrijver, The evolution of sustainable development in international law: inception, meaning and status, 2008 .

${ }^{4}$ Guy Beaucamp, Das Konzept der zukunftsfähigen Entwicklung im Recht, 2002, 151 ff..

${ }^{5}$ Katja Gehne, Legal Enforcement of Sustainable Development within the framework of the EC Treaty (EU Secretariat General Publication, 2000); Mario Prost, Is European Law Becoming More Sustainable?, in : Marie Cordonier Segger, Markus Gehring, Sustainable Development in World Trade Law (2006), 415-459, 2006; vgl. indes Tendenzen in der EU Vergaberechtsprechung, Marc Steiner, Die umweltfreundliche Beschaffung. Vergaberechtliche Möglichkeiten und Grenzen, Gutachten, verfügbar unter http://www.nachhaltige-beschaffung.ch.; Marc Bungenberg/Carsten Nowak, Europäische Umweltverfassung und EG-Vergaberecht - zur Berücksichtigung von Umweltschutzbelangen bei der Zuschlagserteilung, in: ZUR 2003, 10-15.
} 
meinsame europäische Investitionspolitik (Art. 205, 206, 207 AEUV) sieht 21 Abs. 2 (d) auch vor, dass die Europäische Union gemeinsame Politiken und Maßnahmen so zu formulieren hat, dass in den Entwicklungsländern eine Nachhaltige Entwicklung gefördert wird. Dieser Ansatz spiegelt sich bereits in dem 2008 abgeschlossenen Partnerschaftsabkommen zwischen der EU und den CARIFORUM Staaten. Es legt in seinem ersten Grundlagenteil fest, dass es sich um eine "Handelspartnerschaft für Nachhaltige Entwicklung" handelt, wobei Art. 3 Abs. 1 des Abkommens bestimmt, "dass das Ziel der Nachhaltigen Entwicklung auf jeder Ebene der wirtschaftlichen Partnerschaft anzuwenden und zu integrieren ist" ${ }^{\prime \prime}$. Nach Art. 21 Abs. 2 (b) des AEUV sind in der EUAußenpolitik des Weiteren das Demokratie- und Rechtstaatsprinzip, Menschenrechte sowie Prinzipien des Völkerrechts zu berücksichtigen; hierbei handelt es sich um Standards, die ebenfalls zu den Kern-Elementen einer Nachhaltigen Entwicklung gehören.

Inhalt und Aussagegehalt des Nachhaltigkeitskonzepts sind bis heute nicht abschließend geklärt und nicht nur unter Juristen umstritten ${ }^{7}$. Während einige im Recht mit dem Konzept eine rein ökologische Ausrichtung verbinden (nachhaltige Nutzung von natürlichen Ressourcen, Naturschutz für die Nachwelt) ${ }^{8}$, weisen andere auf Prinzipien hin, die im Zusammenhang mit dem Nachhaltigkeitskonzept normative Bedeutung erlangt haben (z.B. das Vorsorgeprinzip, das Partizipationsprinzip, der Schutz natürlicher Ressourcen) 9 . Vielfach wird dem Nachhaltigkeitskonzept auch Inhaltslosigkeit oder Beliebigkeit bescheinigt, da es sich nicht mit einer konkreten inhaltlichen Sollensaussage verbinden lässt, die genaue Anweisungen darüber enthält, welches konkrete Verhalten im Einzelfall das nach dem Nachhaltigkeitskonzept gebotene ist ${ }^{10}$. Ein näherer Blick hinter die Kulissen des Nachhaltigkeitskonzepts, wie es von der Weltkommission für Umwelt und Entwicklung entworfen

\footnotetext{
6 "The objective of sustainable development is to be applied and integrated at every level of their economic partnership...", vgl. Artikel 3 Abs. 1 des EU-CARIFORUM Vertrages. Ähnliche Bestimmungen finden sich bereits in den Vorgängerabkommen, den Verträgen von Lomé und Cotonou, vgl. für einen Überblick http:// trade.ec.europa.eu/doclib/html/138569.htm.

7 Vgl. hierzu noch unten, I, 3.

8 Epiney, Astrid/Scheyli, Martin; Le concept de développement durable en droit international public. In: Schweizerische Zeitschrift für internationales und europäisches Recht (SZIER) 1997, 247-266; Murswiek, Dietrich, "Nachhaltigkeit" - Probleme der rechtlichen Umsetzung eines umweltpolitischen Leitbildes. In: Natur und Recht: Zeitschrift für das gesamte Recht zum Schutze der natürlichen Lebensgrundlagen und der Umwelt, 641-648.

9 Vgl. hierzu die von der International Law Association (ILA) identifizierten KernNachhaltigkeitsprinzipien, Report of the Seventieth Conference, New Delhi (2002), Committee on Legal Aspects of Sustainable Development, 381; Konrad Ginther/Paul J.I.M. de Waart, Sustainable development as a matter of good governance: an introductory view, in: dies. (Hrsg.): Sustainable development and good governance; sowie die Beiträge in Marie-Claire CordonierSegger, Ahsfaq Khalfan (Hrsg.): Sustainable development law: principles, practices and prospects, 2004.

${ }^{10}$ Vgl. zu dieser Diskussion Guy Beaucamp, Das Konzept der zukunftsfähigen Entwicklung, 2002, 72 ff.; Ivo Appel, Staatliche Zukunfts- und Entwicklungsvorsorge, 2005, 27; Gerd Ketteler, Der Begriff der Nachhaltigkeit im Umwelt-und Planungsrecht, in: Natur und Recht 2002, 513521, 515; Angela Schmitz, Sustainable Development: Paradigma oder Leerformel?, in: Dirk Messner/Franz Nuscheler (Hrsg.),Weltkonferenzen und Weltberichte, 1996, 175-185.
} 
wurde und mit dem Aktionsplan Agenda 21 seit dem Weltgipfel für Umwelt und Entwicklung 1992 in Rio Eingang in ungezählte nationale wie internationale Erklärungen, Strategien und Rechtstexte gefunden hat, legt indes ein deutliches normatives Anliegen nahe, das im Folgenden erörtert werden soll (I). So gefasst, und auch von internationalen Spruchkörpern (ICJ, WTOAppellate Body) ähnlich rezipiert, lassen sich aus dem Nachhaltigkeitskonzept rechtliche Leitlinien ableiten, wenn es um die Beurteilung der Legitimität von Investorschutzinteressen und staatlichen Regulierungsinteressen geht (II).

\section{Inhalt und Aussagegehalt des Nachhaltigkeitskonzepts}

\section{Nachhaltigkeit als Effizienzprinzip}

Das Nachhaltigkeitskonzept, wie es sich infolge des Rio-Prozesses heute in Politik und Recht verankert findet, ist ein Kind der entwicklungspolitischen Strategien, die die Vereinten Nationen mit dem Ausruf der Ersten Entwicklungsdekade im Jahre 1960 bis heute erarbeiten, anpassen und weiterentwickeln. Es spiegelt eine konzeptionelle Synthese aus 30 Jahren Entwicklungspolitik mit verschiedenen Strategien, Ansätzen und Erfahrungen ${ }^{11}$. Zwar reichen die Wurzeln des Nachhaltigkeitskonzepts weit tiefer in die politische Philosophie und Geschichte zurück, wo es die verschiedensten Ausprägungen und Definitionen erfahren hat ${ }^{12}$. Im Kontext der Entwicklungsstrategien der Vereinten Nationen hat das Nachhaltigkeitskonzept indes eine spezifische normative Note erhalten, zu der sich die internationale Staatengemeinschaft wiederholt im Zug zahlreicher Weltgipfel, Weltberichte und Aktionspläne sowie in internationalen Vertragswerken bekannt hat. Es ist diese mit dem UN-Kontext verknüpfte inhaltliche Maßgabe des Nachhaltigkeitskonzepts, die heute, verankert über Politiken im Rahmen der Aktionspläne der Konferenzen von Rio und Johannesburg, im nationalen wie internationalen Zusammenhang vorherrscht ${ }^{13}$.

Das hierfür grundlegende Konzept wurde Ende der 1980er Jahre von der Weltkommission für Umwelt und Entwicklung (WCED oder „BrundtlandKommission“) unter dem Vorsitz der damaligen norwegischen Ministerpräsi-

${ }^{11}$ Katja Gehne, Nachhaltige Entwicklung als Rechtsprinzip, normativer Aussagegehalt, rechtstheoretische Einordnung, Funktionen im Recht, im Erscheinen (Mohr-Siebeck 2010), Kapitel 1.

${ }_{12}$ Vgl. Die Beiträge in Wolfgang Kahl (Hrsg.), Nachhaltigkeit als Verbundbegriff, 2008, Edith Brown-Weiss, In fairness to future generations: International law, common patrimony, and intergenerational equity, 1989, 17 ff.; Christopher Gregory Weeramantry, Seperate Opinion ICJGabcikovo-Nagymaros, 25. September 1997, in: International Legal Materials 1998, 204-216, $205 \mathrm{ff}$. .

${ }^{13}$ Der Begriff der Nachhaltigkeit findet sich auch in einem anderem Kontext mit anderem Hintergrund, so z.B. im deutschen Steuerrecht, § 15 II EstG, vgl. hierzu BFH vom 12. Juli 2007, X R 4/04; vgl. zu weiteren Feldern und spezifischen Bedeutungen des Nachhaltigkeitsbegriffs Michael Ronellenfitsch, Umwelt und Verkehr unter dem Einfluss des Nachhaltligkeitsprinzips, in: NVwZ 2006, 385-389, 387; Walter Bückmann/Yeong Heui Lee/Udo E. Simonis, Nachhaltigkeit und das Recht, in: Aus Politik und Zeitgeschichte 2003, 27-32, $30 \mathrm{f}$. 
dentin Gro Harlem Brundtland als „Weg aus der Sackgasse"14 des weltweiten entwicklungspolitischen und ökologischen Missmanagements erarbeitet $^{15}$. Nach der berühmt gewordenen Nachhaltigkeitsformel der BrundtlandKommission definiert sich Nachhaltige Entwicklung als "development that meets the needs of the present without compromising the ability of future generations to meet their own needs". Die Bedeutung dessen erschließt sich, wenn man die Formel im Kontext des gesamten Berichtes und der Entwicklungskonzepte liest, aus denen das Nachhaltigkeitskonzept hervorgegangen ist. Es wird deutlich, dass das Konzept im Wesentlichen verlangt, dass soziale, ökologische und wirtschaftliche Ziele in ihrer Interdependenz erkannt und mit Blick auf langfristigen gesellschaftlichen Wohlstand möglichst effizient gesteuert werden sollen. Eine solche Sichtweise verlangt, Zusammenhänge, Synergie-Effekte und Interessenkongruenzen $\mathrm{zu}$ identifizieren und konsequent zum Wohl sozialer, ökologischer und ökonomischer Ziele zu nutzen (z.B. Investition in Umwelttechnologie, Wirtschaftskapazität durch Bildung und physische wie psychische Gesundheit und Förderung). Hierzu gehört es auch, trade-offs (z.B. Holzenergienutzung auf Kosten des Waldbestandes, Menschenrechtsverletzungen als Nebeneffekte von ausländischen Investitionen) zu identifizieren und möglichst abzumildern oder gering $\mathrm{zu}$ halten sowie im Hinblick auf die Lebenssituation künftiger Generationen gesellschaftliche Kapazitäten und natürliche Ressourcen zu erhalten und zu fördern.

\section{Der normative Referenzrahmen}

Den normativen ${ }^{16}$ Referenzrahmen für die ökonomische, soziale und ökologische Zielrichtung des Nachhaltigkeitskonzepts bilden dabei diejenigen Prinzipien und Standards, die sich in zahlreichen internationalen Erklärungen und Vertragswerken, im Kontext des Nachhaltigkeitskonzepts (Rio-Prozess, verschiedene Weltgipfel) ${ }^{17}$, aber auch in spezifischen mit ökologischen, sozialen oder ökonomischen Standards befassten Abkommen und Erklärungen als normative Maßstäbe und Leitlinien für die internationale Staatengemeinschaft herauskristallisiert haben ${ }^{18}$. Die ökonomische Ziel- und Wert-Komponente ist mit dem Ende des Zweiten Weltkrieges, der Initiative der Havanna Charta, dem letztlich abgeschlossenen GATT und der später gegründeten WTO von Prinzipien der Wirtschaftsfreiheit, der Nichtdiskriminierung sowie des hier-

\footnotetext{
${ }^{14}$ Klaus Töpfer, Sustainable Development, in: Rudolf Dolzer/Josef Thesing (Hrsg.), Protecting our environment - German perspectives on a global challenge, 9-22, 9.

${ }^{15}$ Vgl. World Commission on Environment and Development, Our common future (BrundtlandBericht), Oxford 1987, 40 ff.; vgl hierzu GA Res. 45/199 vom 21. Dezember 1990 - International Develpment Strategy for the Fourth United Nations Development Decade, Präambel.

${ }^{16}$ Der Begriff „normativ“ ist hier nicht im Sinne rechtlicher Normativität und Geltung, sondern im Sinne wertbezogener Normativität (axiologisch) zu begreifen, vgl. zur Unterscheidung von wertbezogenen und rechtlichen Normen Robert Alexy, Theorie der Grundrechte, $1985,79 \mathrm{ff}$. .

${ }^{17}$ Vgl. für eine Einführung und einen Überblick Nico Schrijver, The evolution of sustainable development in international law: inception, meaning and status, 2008.

${ }^{18}$ Vgl. hierzu WTO-Appellate Body, US-Shrimp, WT/DS58/AB/R, 12. Oktober 1998, Rn. 153 ff..
} 
mit verknüpften Investitions- und Eigentumsschutzes geprägt ${ }^{19}$. Die soziale Komponente spiegelt sich in den Bedingungen für eine friedliche Weltordnung, wie sie in der UN-Charta in der Praämbel sowie in Art. 55, 56 der Charta angelegt sind. Hieraus hat sich im Laufe des Bestehens der Vereinten Nationen mit und neben dem Menschenrechtspakt über soziale und wirtschaftliche Rechte, den zuständigen UN-Institutionen zu deren Umsetzung (z.B. ECOSOC, High Commissioner for Human Rights), sowie Internationalen Organisationen, die den Schutz von Menschenrechten zum Gegenstand haben (z.B. ILO, IMO, FAO), ein internationaler Rechtskörper herausgebildet, der teilweise als „internationales Sozialrecht" bezeichnet wird ${ }^{20}$. Zu der sozialen Komponente gehören darüber hinaus als Voraussetzung stabiler politischer Verhältnisse und rationaler, tragfähiger Politikentscheidungen ebenso Prinzipien politischer Ordnung, wie z.B. Demokratie-, bzw. Partizipation, politische Rechte, das Rechtsstaatprinzip, etc., wie sie allgemein mit dem Begriff „Good Governance" verbunden sind ${ }^{21}$. Ähnlich lässt sich die ökologische Komponente des Nachhaltigkeitsbegriffs in diejenigen Prinzipien und Standards fassen, die sich im Zuge der letzten 30 Jahre UN-Umweltpolitik und internationaler Rechtsetzung im Umweltbereich als Leitlinien herausgebildet haben ${ }^{22}$. Hierzu gehören etwa Prinzipien wie das Vorsorgeprinzip, das Verursacherprinzip, der schonende Umgang mit natürlichen Ressourcen, etc.., aber auch Standards, die in Umweltabkommen verankert eine große Mehrheit der Staatengemeinschaft binden.

\section{Die konzeptionelle Funktion und ihre Rezeption}

Das Nachhaltigkeitskonzept hat damit eine doppelte konzeptionelle Funktion: es steht einerseits für die Maßgabe, ökonomische, soziale und ökologische Gesichtspunkte in ihrer Interdependenz wahrzunehmen und ihnen im Hinblick auf die Lage heutiger und künftiger Generationen mittels möglichst effizienter Steuerung zu Gunsten aller Aspekte zu begegnen. Andererseits versammelt das Konzept unter seinem normativen Dach Standards, die Auskunft darüber geben, welche Prinzipien und Leitlinien in diesem Kontext mit Blick auf Aspekte des Gemeinwohls, wie gesellschaftlichen Frieden und Wohlstand sowie den Erhalt von Natur und Ressourcen, zu fördern und zu berücksichtigen sind. Dabei geht es sowohl um das nationale als auch um das internationale

\footnotetext{
${ }^{19}$ Vgl. zum liberalen Wirtschaftsverständnis im Nachhaltigkeitskontext World Commission on Environment and Development (WCED), Our common future (Brundtland-Bericht), Oxford 1987, 49 ff; Erklärung von Rio, Prinzip 12, Agenda 21, Kapitel 30; Daniel Eisermann, Die Politik der Nachhaltigen Entwicklung. Der Rio-Johannesburg-Prozess, Informationszentrum Entwicklungspolitik (IZEP), 2003, 139, verfügbar unter www.inwent.org.

${ }^{20}$ Marie-Claire Cordonier Segger, Ashfaq Khalfan, Salim Nakjavani, Weaving the Rules for Our Common Future: Principles, Practices and Prospects for International Sustainable Development Law. Centre for International Sustainable Development Law (CISDL). Montreal 2002, verfügbar unter: www.cisdl.org/wtr/pdf/WeavingtheRulesOct2002.pdf.

${ }^{21}$ Vgl. die Beiträge in Konrad Ginther/Paul J.I.M. de Waart (Hrsg.): Sustainable development and good governance, 1995.

${ }^{22}$ Wolfgang Kahl, Der Nachhaltigkeitsgrundsatz im System der Prinzipien des Umweltrechts, in: Hartmut Bauer/Detlef Czybulka/Wolfgang Kahl/ Andreas Voßkuhle (Hrsg.), Umwelt, Wirtschaft und Recht, Tübingen 2002, 111-148.
} 
Gemeinwohl. Die Brundtland-Kommission unterstreicht, dass sich das Nachhaltigkeitskonzept an alle Länder und Regierungsebenen richtet, ungeachtet ihres Entwicklungsstandes. Die Nachhaltigkeitsverantwortung greift grundsätzlich dort, wo gesellschaftliche Prozesse politisch gesteuert und rechtlich geregelt (Regierungsfunktion) oder auch beeinflusst (Unternehmen) werden ${ }^{23}$. Die Staatengemeinschaft wird hierbei als interdependente Entität konzipiert, die in zentralen, das Überleben und die Zukunft der Menschheit betreffenden Fragen (Umwelt-, Ressourcen-, Klimaschutz, Friedenssicherung, Armut, Bevölkerungsexplosion, Wirtschaftsbeziehungen, Terrorismusbekämpfung) auf Kooperation, Koordination und gegenseitige Rücksichtnahme angewiesen ist ${ }^{24}$. Innerhalb dieser internationalen Entität sei es an jeder politischen Einheit, im Rahmen der international abgestimmten Leitlinien spezifische Nachhaltigkeitsstrategien und Standards je nach Kultur- und Entwicklungsstand über partizipative und integrative Entscheidungsprozesse zu prägen ${ }^{25}$. Die heute weltweit zahlreichen nationalen wie internationalen Strategien und Regelungswerke im Nachhaltigkeitskontext spiegeln diesen Aufruf wider.

Die Verwirrung, die vielfach mit den Inhalten des Nachhaltigkeitsbegriffs einhergeht, dürfte vor allem darauf zurückzuführen sein, dass die Frage, ob und wie dem steuerungspolitischen Effizienzanspruch des Nachhaltigkeitskonzepts genüge getan werden kann und soll, in den letzten Jahrzehnten Gegenstand lebhafter, Disziplinen übergreifender Diskussion und Meinungsverschiedenheiten war. Unter den vielen Facetten lassen sich grundsätzlich drei Hauptrichtungen erkennen: Erstens, die Priorität von Wachstum und Markt, die dem Faktor des Wirtschaftswachstums einen übergeordneten Stellenwert einräumt. Hier geht man davon aus, dass ökologische und soziale Faktoren vor allem dann profitieren, wenn ein möglichst ausgeprägtes Wirtschaftswachstum erzielt wird (Kuznet Curve, Substitutionsparadigma). Über die dadurch generierte Nachfrage nach gesellschaftlichen Gütern sowie Anreizen und Mitteln für die Entwicklung von Technologien gewinne die Gesellschaft Kapazitäten im Umgang mit sozialen und ökologischen Fragestellungen, auch künftiger Generationen ${ }^{26}$. Mit der Annahme, dass effiziente Regulierung im politischen Kontext nicht möglich sei, werden nicht regulierte Marktprozesse als Garant für eine effiziente Allokation von Ressourcen verstanden (vgl. z.B.

\footnotetext{
${ }^{23} \mathrm{Vgl}$. die Arbeit des Special Representative of the Secretary-General on human rights and transnational corporations and other business enterprises (John Ruggie), Informationen und Berichte unter http:// www2.ohchr.org/english/issues/trans_corporations/index.htm; Agenda 21, Kapitel 30.

${ }^{24}$ World Commission on Environment and Development, Our common future (BrundtlandBericht), Oxford 1987, 40 ff..

${ }^{25}$ World Commission on Environment and Development, Our common future (BrundtlandBericht), Oxford 1987, 49 ff..

26 Vgl. für einen Überblick m.w.N. Sachverständigenrat für Umweltfragen, Umweltgutachten 2002, 64 ff; Bas Arts, Nachhaltige Entwicklung: eine begriffliche Abgrenzung, in: Peripherie 1994, 12 ff., Joachim H. Spangenberg, Die ökonomische Nachhaltigkeit der Wirtschaft, 61 ff.; Fred Luks, Die Zukunft des Wachstums, 2001, 31 ff..
} 
Chicago School27). Zweitens ist im Zusammenhang mit dem Nachhaltigkeitskonzept ein ökologisches Paradigma zu beobachten, das Umwelt- und Ressourcenschutz im Nachhaltigkeitskontext als maßgebliche Größe betrachtet. Auf der Basis von Ressourcenknappheit sei weder Wachstum noch gesellschaftlicher Frieden und Wohlstand denkbar, daher müsse dem Umwelt- und Ressourcenschutz im Spannungsverhältnis zwischen wirtschaftlichen und sozialen Interessen grundsätzlich Priorität eingeräumt werden ${ }^{28}$. Drittens schließlich verstehen einige das Nachhaltigkeitskonzept als EffizienzParadigma, das staatliche Steuerungsprozesse vor die Herausforderung stellt, kurz- und langfristigen Zielen im Spannungsfeld zwischen ökologischen, ökonomischen und gesellschaftlichen Interessen durch effiziente Steuerungsprozesse zu begegnen; dies über die Integration von Politikfeldern und das systematische Aufdecken und Abfedern von trade-offs und Nutzen von Synergieeffekten im Nachhaltigkeitskontext ${ }^{29}$.

Das ökonomische Paradigma (Wachstum zuerst, Nachhaltigkeit später) hat im öffentlichen Diskurs und in der Politik bisher tendenziell die Überhand behalten, was sich nicht zuletzt in einer bis heute primär auf Investitionsschutz ausgerichteten internationalen Investitionspolitik für Wachstum und Entwicklung widerspiegeln dürfte ${ }^{30}$. Es bestand und besteht die Tendenz, Nachhaltigkeit in die „drei Säulen“ (Ökonomie, Umwelt, Gesellschaft) aufzuteilen und jede Säule als Herausforderung getrennt zu betrachten. Nicht zuletzt vor dem Hintergrund der internationalen Finanzkrise und der Bedrohung durch den Klimawandel ist jedoch die Tendenz zu spüren, von einem primär auf Markt- und Wachstumspolitik vertrauenden Denken zu einem Ansatz überzugehen, der die gesellschaftliche und ökologische Perspektive über effiziente Regelung einbezieht ${ }^{31}$. Auch Unternehmen betrachten heute Menschenrechte, Entwicklungsfragen und ökologische Herausforderungen mehr und mehr als „busi-

\footnotetext{
${ }^{27}$ Milton Friedman, Capitalism and freedom, 1962, 22 ff; Milton Friedman/Rose Friedman: Free to Choose: The Classic Inquiry into the Relationship between Freedom and Economics, 1973, 27 ff..

28 Dietrich Murswiek, "Nachhaltigkeit" - Probleme der rechtlichen Umsetzung eines umweltpolitischen Leitbildes, in: Natur und Recht 2002, 643; Astrid Epiney/Martin Scheyli, Strukturprinzipien des Umweltvölkerrechts, 1998, 44, 60 ff.; Herman E. Daly, Beyond growth, the economics of sustainable development, 1996.

${ }^{29}$ So z.B. über die gezielte Einbindung von Unternehmen, die Ausrichtung von Unternehmensinteressen auf sozial-ökologische Fragestellungen, oder auch Nachhaltigkeitsprüfungen, die Interdependenzen und Synergieeffekte aufdecken und flankierende Maßnahmen zur Abfederung negativer Auswirkungen prüfen, vgl. z.B. die Nachhaltigkeitsprüfungen im Rahmen der EU Markus Gehring, Nachhaltigkeit durch Verfahren im Welthandelsrecht: Umwelt- und Nachhaltigkeitsprüfungen und die WTO, 2007; Armin Grunwald, Jürgen Kopfmüller, Die Nachhaltigkeitsprüfung: Kernelement einer angemessenen Umsetzung des Nachhaltigkeitsleitbilds in Politik und Recht, 2007.

${ }^{30}$ Roberto Echandi, What do developing countries expect from the international investment regime, Working Paper, 2008; Karl Georg Zinn, Die Selbstzerstörung der Wachstumsgesellschaft. Politisches Handeln im ökonomischen System, 1980.

${ }^{31}$ Edward Balleisen, David Moss, "Introduction", in: dies. (Hrsg.), Government and Markets: Toward a New Theory of Regulation, 2009; Commission on the Measurement Of Economic Performance and Social Progress, Joseph Stiglitz, Amartya Sen, Kenneth Arrow, and others, verfügbar unter: http:/ / www.stiglitz-sen-fitoussi.fr/en/membres.htm.
} 
ness case“. Sie sehen sie nicht nur als Wirtschaftspotential (Investitionsfonds, Umweltgüter), sondern integrieren diese Faktoren auch in ihre Risikoanalysen $^{32}$. Blickt man aus dieser Perspektive auf den Kontext des internationalen Investitionsrechts, dürfte dies nicht ohne Konsequenzen für dessen Anwendung und Ausgestaltung bleiben: Das rechtliche Vorverständnis, mit dem man an investitionsrechtliche Fragestellungen herantritt, lässt sich vor diesem Hintergrund kaum mehr auf den Schutz und die Förderung von Investitionen reduzieren. Will es dem Aspekt einer gesamtgesellschaftlichen Effizienz gerecht werden, gilt es, den Fokus zu erweitern, um rechtliche Mechanismen zu entwickeln, die es erlauben, vor diesem Hintergrund Investorinteressen und staatliche Maßnahmen angemessen und rechtlich vorhersehbar $\mathrm{zu}$ beurteilen ${ }^{33}$.

\section{Die rechtliche Bedeutung des Nachhaltigkeitskonzepts}

\section{Der rechtliche Aussagegehalt des Nachhaltigkeitskonzepts}

Begreift man das Nachhaltigkeitskonzept nach dem oben skizzierten Aussagegehalt als eine an Entscheidungsträger gerichtete normative Vorgabe, so stellt es eine Regel auf: „Handele so, dass Deine Politiken im Hinblick auf soziale, ökonomische und ökologische Belange sowie mit Blick auf kurzfristige und langfristige Interessen möglichst effizient sind“34. Diese Vorgabe wird vielfach als für den rechtlichen Zusammenhang untauglich klassifiziert, da sie keine konkrete Maßgabe an die Hand gebe, die dem Entscheidungsträger vermittele, was konkret im Einzelfall eine mit dem Nachhaltigkeitskonzept vereinbare Maßnahme sei. Aufgrund der vielen gegenläufigen Möglichkeiten, Maßnahmen mit normativen Aspekten des Nachhaltigkeitskonzepts zu verknüpfen, sei die Aussage des Nachhaltigkeitskonzepts nahezu beliebig ${ }^{35}$. Diese Meinung spiegelt ein strenges positivistisches Rechtsverständnis, das mit der heutigen Rechtsrealität, wie sie etwa mit dem deutschen Verfassungsrecht oder auch der Rechtspraxis im europarechtlichen und internationalen Kontext

\footnotetext{
${ }^{2}$ Vgl. z.B. die Finanzierungsleitlinien der International Financial Cooperation (World Bank), http://www.ifc.org/ifcext/sustainability.nsf/Content/OurStories_SocialResponsibility_Hum anRights.

${ }^{33}$ Investitionsstreitigkeiten lassen sich heute kaum mehr auf einen rein privatrechtlichen Kontext reduzieren Das weit gespannte Netz von über 2600 bilateralen und regionalen IIA hat nicht nur zu einer „Explosion“ von Investitionsstreitigkeiten geführt. Es hat auch zur Folge, dass Investitionsstreitigkeiten mehr und mehr die Überprüfung staatlicher Regelungen im öffentlichen Interesse zum Gegenstand haben, vgl. Gus Van Harten, Martin Loughlin, Investment Treaty Arbitration as a Species of Global Administrative Law, in: The European Journal of International Law 2006, 121-150.

${ }^{34}$ Katja Gehne, Nachhaltige Entwicklung als Rechtsprinzip, normativer Aussagegehalt, rechtstheoretische Einordnung, Funktionen im Recht, Fn. 11, Kapitel 2.

${ }^{35}$ Walter Bückmann, Probleme der Transformation des Nachhaltigkeitsgebots in das Recht, in: Karl-Werner Brand (Hrsg.): Politik der Nachhaltigkeit, 2002, 145 f.; Guido Leidig, Nachhaltigkeit als umweltplanerisches Entscheidungskriterium, in: Zeitschrift für Umwelt- und Planungsrecht 2000, $371 \mathrm{ff}$. .
} 
kaum vereinbar ist ${ }^{36}$. Die Rechtsanwendung ist von Prinzipien und unbestimmten Rechtsbegriffen geprägt, für deren Auslegung auf den rechtlichen Kontext oder auf ein „lex generalis“ zurückgegriffen wird, ohne dass dessen Inhalt für den Einzelfall normativ konkret festgelegt wäre ${ }^{37}$. In vielen Rechtsordnungen spielt der Grundsatz der Verhältnismäßigkeit eine wichtige Rolle, in dessen Kontext Rechtspositionen anhand von Maßgaben der komparativen Notwendigkeit und Angemessenheit beurteilt und abgewogen werden. Diese rechtliche Leitlinie ist ebenfalls weit von einer konkreten, normativen Handlungsmaßgabe entfernt und wird doch in ihrem Rechtspflichtgehalt (Pflicht des verhältnismäßigen Handelns) heute kaum mehr in Frage gestellt ${ }^{38}$. Die rechtliche Beurteilung erfolgt hier in Verknüpfung mit einer der Rechtsordnung zugrunde liegenden normativen Tiefenstruktur des Rechts, die eine Matrix aus generellen Prinzipien, Grund- und Menschenrechten oder auch Zielen der Rechtsordnung an die Hand gibt ${ }^{39}$. Solcher Art Rechtsanwendungsprinzipien, wie die Verhältnismäßigkeitsprüfung, die verfassungskonforme Auslegung oder auch Artikel 31 Abs. 1 WVK, in Verbindung mit einer relativ bestimmbaren Tiefenstruktur der jeweiligen Rechtsordnung (Verfassungsordnung, allgemeines Völkerrecht, Vertragsziele), fassen die Unbestimmtheit in einen flexiblen, aber in seiner Zielrichtung klaren, Rechtsrahmen ${ }^{40}$.

Im Hinblick auf das Nachhaltigkeitskonzept verhält es sich ähnlich. Seine Verhaltensmaßgabe gibt eine normative Struktur vor: möglichst effizient soziale, ökologische, ökonomische und zukunftsbezogene Belange einbeziehen bedeutet zunächst, sich ein umfassendes Bild der zu regelnden oder zu steuernden Situation zu verschaffen (Integrationsprinzip), und ein Mittel zu wählen, das im Spannungsverhältnis dieser Belange möglichst effizient und angemessen ist (Mittelerhebung und Wertabwägung). Die hierfür erforderlichen Informationen können über Nachhaltigkeitsprüfungen erhoben und über partizipative, integrative Entscheidungsprozesse vertieft werden ${ }^{41}$. Die Besonderheit des Nachhaltigkeitskonzepts besteht dabei darin, dass es, unabhängig von der Rechtsordnung, in der es Anwendung findet, seine spezifische normative Tiefenmatrix mitbringt, nämlich den Kontext derjenigen Prinzipien und Standards, die im sozialen, ökonomischen und ökologischen Kontext und im Hinblick auf das Nachhaltigkeitskonzept im internationalen Kontext geprägt worden sind ${ }^{42}$. Das Nachhaltigkeitskonzept stellt hier, wie z.B. auch das Rechts-

\footnotetext{
${ }^{36}$ Katja Gehne, Nachhaltige Entwicklung als Rechtsprinzip, Fn. 11, Kapitel 4, IV.

${ }^{37}$ Joost Pauwelyn, Bridging fragmentation and unity: international law as a universe of interconnected islands, in: Michigan Journal of International Law, 2004, 905-916; Ulrich Karpenstein, Praxis des EG-Rechts : Anwendung und Durchsetzung des Gemeinschaftsrechts in der Bundesrepublik Deutschland, 2006.

${ }^{38}$ Oliver Koch, Der Grundsatz der Verhältnismäßigkeit in der Rechtsprechung des Gerichtshofs der Europäischen Gemeinschaften, 2003.

${ }^{39}$ Klaus F. Röhl, Allgemeine Rechtslehre: ein Lehrbuch, 2001, 603.

${ }^{40}$ Vgl. zu der normativen Dichte und rechtlichen Vorhersehbarkeit dieser Art Rechtstechnik Robert Alexy, Rechtssystem und praktische Vernunft, in: Rechtstheorie 1987, 405-419, 414.

${ }^{41}$ Katja Gehne, Nachhaltigkeit als Rechtsprinzip, Fn. 11, Kapitel 2.

${ }^{42}$ Vgl. hierzu die Ausführungen oben zum normativen Referenzrahmen des Nachhaltigkeitskonzepts (I, 2.), zu den Kern-Prinzipien des Nachhaltigkeitskonzepts Nico Schrijver, The Evo-
} 
staatsprinzip oder das Demokratieprinzip, eine Standard-Norm dar, deren Inhalt durch ein Konglomerat verschiedener Prinzipien und Standards konkretisiert wird ${ }^{43}$. Darüber hinaus greift im Nachhaltigkeitskontext auch in Verbindung mit dem Nachhaltigkeitskonzept die für die Wertabwägung maßgebliche normative Ordnung der jeweils einschlägigen Rechtsordnung und deren ökonomisch, sozial und ökologisch relevante Standards (im Völkerrecht wären dies z.B. die Teilbereiche des Umweltvölkerrechts, des internationalen Wirtschaftsrechts, des internationalen Sozialrechts und die damit verbundenen Normen, Ziele und Grundsätze). Im Hinblick auf die Rechtskontrolle im Zusammenhang mit dem Nachhaltigkeitskonzept geht es dann darum zu überprüfen, inwieweit Entscheidungsträger alle betroffenen Belange einbezogen haben, effiziente Steuerungsmittel gewählt worden sind, welche die Belange so weit wie möglich berücksichtigen und inwieweit sich die Wahl des Mittels im Hinblick auf das Regelungsziel in den Grenzen der angemessenen Belastung von Rechtspositionen und öffentlichen Interessen bewegt. Eine ähnliche rechtliche Überprüfung des Nachhaltigkeitskonzepts erfolgt heute z.B. im deutschen Baurecht im Zusammenhang mit $\S 1$ Abs. 5 des Baugesetzbuches (Grundsätze der Bauleitplanung)44. Inwieweit aus rechtlicher Perspektive Gerichte oder Entscheidungskörper über die Angemessenheit einer Maßnahme im Nachhaltigkeitskontext befinden können, hängt davon ab, bis zu welchem Grad politische Entscheidungen in einer Rechtsordnung der gerichtlichen Kontrolle unterliegen ${ }^{45}$. In vielen Rechtsordnungen genießen Entscheidungsträger insbesondere auf Gesetz gebender Ebene einen erheblichen Entscheidungsspielraum (judicial self restraint) ${ }^{46}$. Im WTO-Recht hat der Appellate Body z.B. mehrfach anerkannt, dass es den Mitgliedstaaten vorbehalten sei, im Rahmen der Ausnahmebestimmungen das für sie angemessene Schutzniveau $\mathrm{zu}$ bestimmen ${ }^{47}$

lultion of Sustainable Development in International Law: Inception, Meaning and Status, 2008, 208.

${ }^{43}$ Vgl. zu der Normform des Standards und dessen Bedeutung im internationalen Kontext Eibe Riedel, Standards and sources. Farewell to the exclusivity of the sources triad in international law? in: European Journal of International Law (EJIL) 1991, 58-84.

${ }^{44}$ Vgl. hierzu Katja Gehne, Nachhaltigkeit als Rechtsprinzip, Fn. 11, Kapitel 5, IV.

${ }^{45}$ Ralph Alexander Lorz, Interorganrespekt im Verfassungsrecht, Funktionenzuordnung, Rücksichtnahmegebote und Kooperationsverpflichtungen; eine rechtsvergleichende Analyse anhand der Verfassungssysteme der Bundesrepublik Deutschland, der Europäischen Union und der Vereinigten Staaten. 2001. 424

${ }^{46}$ Vgl. die Auswirkungen der gerichtlichen Kontrollkompetenz auf die Tragweite des Nachhaltigkeitskonzepts, Katja Gehne, Nachhaltigkeit als Rechtsprinzip, Fn. 11, Kapitel 6.

${ }^{47}$ Vgl. WTO-Appellate Body Report, Korea - Various Measures on Beef, WT/DS161/AB/R; WT/DS169/AB/R, 11. Dezember 2000 Rn. 176; WTO-Appellate Body Report, EC - Asbestos, WT/DS135/AB/R, 12. März 2001, Rn. 168; der Vorbehalt findet sich auch verankert in Abs. 6 der Präambel zum SPS Abkommen: „[...] without requiring Members to change their appropriate level of protection of human, animal or plant life or health", in Abs. 4 der Präambel zum GATS Abkommen: "Recognizing the right of Members to regulate, and to introduce new regulations, on the supply of services within their territories in order to meet national policy objectives [...]"; vgl. Elisabeth Türk, Jan Neumann, Necessity revisited: proportionality in World Trade Organization law after Korea-Beef, EC-Asbestos and EC-Sardines, in: Journal of world trade 2003, 199-233. 
Nichtsdestotrotz bleibt hier Raum, eine offensichtliche Nicht-Angemessenheit einer Maßnahme festzustellen, wenn diese mit dem Prinzip von Treu und Glauben als einem der ältesten und zentralsten Prinzipien des Rechts, nicht zu vereinbaren ist ${ }^{48}$. So z.B., wenn die Maßnahme nicht geeignet ist, das Schutzinteresse sicherzustellen oder, wenn die Maßnahme offensichtlich außer Verhältnis zum Schutzinteresse steht (z.B. Importverbot mit hohem wirtschaftlichen Verlust bei sehr geringer Wahrscheinlichkeit der Einschleppung einer Pflanzenkrankheit ${ }^{49}$ ), wenn offensichtlich entgegen ein von der Rechtsordnung vorgegebenes Ziel (z.B. Umweltschutz) oder auch widersprüchlich (estoppel, venire contra factum proprium) gehandelt wird. In diesem Sinne könnte etwa eine Maßnahme für mit dem Nachhaltigkeitskonzept nicht vereinbar betrachtet werden, wenn zentrale ökonomische, soziale, ökologische oder zukunftbezogene Aspekte außer Acht bleiben oder die Maßnahme diese so wenig einbezieht, dass sie in ihrer Bedeutung untergehen.

\section{Praktische Auswirkungen in der Rechtsanwendung}

Ist das Nachhaltigkeitskonzept in einem internationalen Abkommen (z.B. IIA) verankert oder nimmt man mit dem IGH an, dass dem Nachhaltigkeitskonzept völkerrechtliche Bedeutung zukommt, lassen sich aus dessen oben skizzierter normativer Vorgabe für die Rechtsanwendung bestimmte rechtliche Konsequenzen ableiten. Illustrativ sind hier aus dem internationalen Kontext insbesondere die grundlegenden Entscheidungen des IGH im GabcikovoNagymaros Fall, sowie des WTO Appellate Body im Fall US-Shrimp ${ }^{50}$. In der Konsequenz, wie sie in den Entscheidungen zum Ausdruck kommt, ist mit dem Nachhaltigkeitskonzept im Recht als "Rechtskonzept", wie beide Entscheidungskörper betonen ${ }^{51}$, insbesondere zweierlei verbunden: einerseits die Pflicht der Staaten, eine "angemessene" Lösung im Konflikt zwischen Umwelt- und Entwicklungsbelangen $\mathrm{zu}$ finden ${ }^{52}$. Andererseits ein "Meta-

\footnotetext{
${ }^{48}$ WTO-Appellate Body, US-Shrimp, WT/DS58/AB/R, 12. Oktober 1998, Rn. 158; Christopher R. Rossi, Equity and International Law: A Legal Realist Approach to the Process of International Decisionmaking, 1993.

${ }^{49}$ WTO Appellate Body Report, Japan - Measures Affecting the Importation of Apples, WT/DS245/AB/R, 10. Dezember 2003, Rn. 162 ff..

${ }^{50}$ International Court of Justice, Gabcikovo-Nagymaros, 25. September 1997, in: International Legal Materials 1998, 162 ff., Rn. 140; WTO-Appellate Body, US-Shrimp, WT/DS58/AB/R, 12. Oktober 1998, Rn. 129.

${ }^{51}$ Beide Entscheidungskörper sehen das Nachhaltigkeitskonzept ausdrücklich nicht als Rechtsprinzip, sondern als Rechtskonzept, was zwar die geringe normative Dichte indizieren mag, für die Konsequenz der rechtlichen Aussage indes kaum eine Rolle spielt, vgl. ICJ, Gabcikovo-Nagymaros, 25. September 1997, in: International Legal Materials 1998, 162 ff., Rn. 140; WTO-Appellate Body, US-Shrimp, WT/DS58/AB/R, 12. Oktober 1998, Rn. 129; vgl. hierzu Katja Gehne, Nachhaltigkeit als Rechtsprinzip, Fn. 11, Kapitel 7.

52 ICJ, Gabcikovo-Nagymaros, 25. September 1997, in: International Legal Materials 1998, 162 ff., Rn. 140 ff., Rn. 142; Alan Boyle, The Gabcikovo-Nagymaros Case: New Wine Old Bottles?, in: YIEL 1997, 13-20, 18; Matthew Stilwell, Trade and environment in the context of sustainable development, in: Markus W. Gehring/Marie-Claire Cordonier Segger: Sustainable development in world trade law, 27-72, 2005, 37 ff.; Philippe Sands, International Courts and the Application of the Concept of "Sustainable Development", in: Max Planck United Nations Yearbook 1999, 389-405, $393 \mathrm{f}$.
} 
Prinzip", das als Auslegungsmaxime die Rechtsauslegung in eine bestimmte „Farbe, Textur und Schattierung" taucht ${ }^{53}$, die die Grenzen der Rechtspflichten in unbestimmten Auslegungsbereichen normativ beleuchtet ${ }^{54}$.

Im Fall des Gabcikovo Nagymaros Projektes ging es um einen Streit zwischen Ungarn und der Slowakei über den Bau von zwei Staudämmen, die gemeinsam betrieben werden sollten. Ungarn brachte nach Vertragsschluss Bedenken bezüglich der Artenvielfalt und des Wasserreservoirs ein und kündigte den Vertrag einseitig. Der IGH entschied, dass aus den wissenschaftlichen Berichten, welche die Parteien dem Gerichtshof vorgelegt hatten, eindeutig hervorgehe, dass Umweltschutz in jedem Fall ein Schlüsselaspekt des Projektes sei. In den letzten Jahrzehnten hätten sich mit einer wachsenden ökologischen Verantwortung für heutige und künftige Generationen neue Normen und Standards herausgebildet ${ }^{55}$, die von den Vertragsparteien mit angemessenem Gewicht berücksichtigt werden müssten. Der Aspekt, dass ökonomische Entwicklung mit Umweltschutzaspekten zum Ausgleich zu bringen ist, komme in dem Konzept der Nachhaltigen Entwicklung zum Ausdruck. Hieraus leitet der IGH die Verpflichtung der Parteien ab, über die Umweltschutzfragen, die mit dem Betrieb des Gabcikovo Kraftwerkes verbunden ist, eine Einigung zu erzielen. Das Prinzip von Treu und Glauben nach Art. 26 der WVK verlange von den Parteien, eine angemessene Lösung im kooperativen Rahmen des Vertrages zu erarbeiten. Dabei müssten die Verhandlungen bedeutsam („meaningful“") sein, was nicht der Fall sei, wenn beide auf ihren Positionen beharrten $^{56}$. Es sei nicht an dem Gerichtshof zu entscheiden, wie die Lösung im Einzelnen auszusehen habe. Die Parteien müssten jedoch den Zielen des Vertrages genüge tun und die Normen des Umweltvölkerrechts sowie die Prinzipien im Bereich des internationalen Wasserrechts angemessen berücksichtigen ${ }^{57}$. Mit dem Nachhaltigkeitskonzept sind danach zwei normative Aspekte verbunden: die Pflicht der Parteien, im Rahmen von Treu und Glauben eine Lösung für den offensichtlich tangierten Umweltschutzaspekt zu finden, sowie die Pflicht, dabei die Ziele des Vertrages und die Prinzipien des internationalen Umweltrechts zu beachten. Judge Weeramantry ergänzt in seiner Separate Opinion, dass die Pflicht zum „angemessenen Ausgleich“ im Nachhaltigkeitskontext ebenfalls die Belange des Rechts auf Entwicklung betreffe, d.h. auch den Ausgleich zwischen ökonomischen und entwicklungspolitischen Belangen erfordere ${ }^{58}$. Dabei legt er ausführlich dar, dass das Nachhaltigkeitskonzept

\footnotetext{
53 WTO-Appellate Body, US-Shrimp, WT/DS58/AB/R, 12. Oktober 1998, Rn. 152.

${ }^{54}$ Vaughan Lowe, Sustainable development and unsustainable arguments, in: Alan

Boyle/David Freestone (Hrsg.): International law and sustainable development, 1999, 19-37, $31 \mathrm{ff} .$.

${ }^{55}$ ICJ, Gabcikovo-Nagymaros, 25. September 1997, in: International Legal Materials 1998, 162 ff., Rn. 140.

${ }^{56}$ Ebenda, Rn. $141 \mathrm{ff}$. .

${ }^{57} \mathrm{Vgl}$. Philippe Sands, International Courts and the Application of the Concept of "Sustainable Development", in: Max Planck United Nations Yearbook 1999, 389-405, 393 ff..

${ }^{58} \mathrm{Vgl}$. zu den normativen Inhalten des Rechts auf Entwicklung im Nachhaltigkeitskontext, die ihren Ausdruck vor allem in den Menschenrechten finden, Nico Schrijver, The Evolultion of Sustainable Development in International Law: Inception, Meaning and Status, 2008, S. 77 ff..
} 
in dieser Gestalt heute zu den allgemeinen Prinzipien des Völkerrechts gezählt werden kann ${ }^{59}$.

Dem Fall US-Shrimp vor dem WTO Appellate Body lag eine US-ShrimpFangbestimmung mit extraterritorialer Wirkung zum Schutz von Meeresschildkröten zugrunde. Der Appellate Body hatte zu entscheiden, inwieweit diese in den Anwendungsbereich des Art. XX GATT fällt und den Anforderungen des Chapeau des Art. XX GATT genügt. Der Appellate Body betont in seiner Entscheidung, dass das Nachhaltigkeitskonzept als Präambelbestimmung des WTO-Abkommens eine Auslegungsmaxime darstelle, "that must add colour, texture and shading to our interpretation of the agreements annexed to the WTO Agreement" ${ }^{\prime 60}$. Ähnlich wie der IGH und vor allem Judge Weeramantry in seiner Separate Opinion, betrachtet er das Konzept der Nachhaltigen Entwicklung als ein weithin anerkanntes Konzept, das verlangt, soziale und ökonomische Entwicklung und Umweltschutz $\mathrm{zu}$ integrieren ${ }^{61}$. In diesem Licht und unter Heranziehung relevanter Abkommen im Umweltbereich, darunter insbesondere die Artenvielfaltskonvention, die Agenda 21, die Konvention über migrierende wilde Tierarten und die Konvention über den internationalen Handel mit bedrohten Tier- und Pflanzenarten (CITES), legt der Appellate Body Art. XX (g) GATT dahingehend aus, dass unter den Begriff der "exhaustible natural resources" nicht nur Mineralien und andere nicht lebende Ressourcen fallen, sondern auch vom Aussterben bedrohte biologische Ressourcen der Flora und Fauna. Der Begriff der "natural resources“, der noch aus Zeiten des GATT 1947 stamme, sei nicht statisch, sondern evolutionär zu verstehen. Hier argumentiert der Appellate Body ähnlich wie der IGH, der im Gabcikovo-Nagymaros-Fall ebenfalls angenommen hatte, dass man neu herausgebildete Prinzipien im Völkerrecht berücksichtigen müsse, auch wenn sie sich auf Verträge aus der Vergangenheit beziehen ${ }^{62}$. Im Zuge der Prüfung, inwieweit die Handel beschränkende US-Shrimp-Fangbestimmung nach dem "Chapeau" des Art. XX GATT Bestand haben kann, weist der Appellate Body erneut auf die wichtige Funktion der Präambel und des Nachhaltigkeitsziels sowie die allgemeinen Völkerrechtsprinzipien als Leitlinien der Rechtsauslegung im WTO-Kontext hin ${ }^{63}$. Auf dieser Grundlage wertet er die US-ShrimpFangbestimmung wegen ungenügender Bemühungen der USA um multilaterale Lösungen und mangelnder Flexibilität der Schutzbestimmungen in der Shrimp-Fang-Frage als „unjustifiable“ und „arbitrary discrimination"64. Auch

\footnotetext{
${ }^{59}$ Vgl. Christopher Gregory Weeramantry, Seperate Opinion ICJ-Gabcikovo-Nagymaros, 25. September 1997, in: International Legal Materials 1998, 204-216, 208 ff.; Reinhard Bartholomäi, Sustainable Development und Völkerrecht, der das Nachhaltigkeitskonzept in der Staatenpraxis nachweist, 1997, 72 ff.; Alan Boyle/David Freestone: Introduction, in: dies. (Hrsg.): International law and sustainable development, 1999, 6; a.A und m.w.N. Bernhard Braune, Rechtsfragen der nachhaltigen Entwicklung im Völkerrecht, 2004, 82 ff..

60 WTO-Appellate Body, US-Shrimp, WT/DS58/AB/R, 12. Oktober 1998, Rn. 153.

${ }^{61}$ Ebenda, Rn. 129, Fn. 107.

62 Ebenda, Rn. 129.

${ }^{63}$ Ebenda, Rn. 155.

${ }^{64}$ Ebenda, Rn. 161 ff.; vgl. hierzu jedoch die Gegenargumente mit unterschiedlicher Wertung der amici curiae, Centre of Internernational Environmental Law (CIEL), Amicus Brief to the Ap-
} 
hier bewegt sich der Appellate Body, wenn auch auf anderer Grundlage ${ }^{65}$, in einem ähnlichen Fahrwasser wie der IGH, der den Vertragsparteien im Rahmen des Art. 26 WVK die Pflicht zu Vertragsverhandlungen für eine angemessene Lösung im Nachhaltigkeitskontext aufgab. Die mangelnde Flexibilität der Vertragsbestimmungen, die der Appellate Body als "arbitrary discrimination" klassifiziert, widerspricht dem Nachhaltigkeitskonzept, das im Spannungsverhältnis von Umwelt- und Wirtschaftsinteressen angemessene Lösungen verlangt. Kann dasselbe Schutzniveau auch mit anderen Mitteln mit weniger trade-off Potential für Handel, Umwelt und Entwicklung erreicht werden, sind solche Maßnahmen aber nicht zugelassen, entbehrt dies einer rationalen Grundlage. In der Nachhaltigkeitslogik könnte ein Industriestaat auch im Rahmen des Prinzips der gemeinsamen, aber unterschiedlichen Verantwortung flankierende Maßnahmen ergreifen, z.B. über Entwicklungsfinanzierung, Technologietransfer oder einen Fonds für die Ausrüstung von Shrimpfangschiffen $^{66}$. Am Fall US-Shrimp wird deutlich, dass der Appellate Body nicht das Artenschutzziel für unangemessen klassifiziert, sondern die Art und Weise, wie die USA hiervon entgegen dem Prinzip von Treu und Glauben (good faith) Gebrauch machen. Deshalb verlangt er eine entsprechende Anpassung der Bestimmungen.

\section{Anwendungsperspektiven im internationalen Investitionsrecht}

Geht es darum, Anliegen des Investorschutzes und legitime staatliche Regulierungsinteressen in rechtliche Kriterien $\mathrm{zu}$ fassen, ist zunächst festzustellen, dass das Nachhaltigkeitskonzept mit der Anforderung eines angemessenen Ausgleichs zwischen sozialen (Menschenrechte, Good Governance und Gerechtigkeitsprinzipien), ökologischen (Umwelt- und Ressourcenschutz) und ökonomischen (Prinzipien moderner liberaler Ökonomik) normativen Anliegen gerade auf diese Fragestellung abzielt. Investorschutzinteressen als Ausdruck makroökonomischer Interessen des Staates und staatliche Regulierungsinteressen verknüpft mit sozialen und ökologischen Anliegen eröffnen das Spannungsfeld, auf das sich die normativen Inhalte des Nachhaltigkeitskonzepts beziehen. Die rechtliche Beurteilung des legitimen Investor- bzw. Regulierungsinteresses fällt insofern in den Anwendungsbereich des Nachhaltigkeitskonzepts. Nach dem oben Gesagten, sind mit dem Nachhaltigkeitskonzept grundsätzlich zwei normative Vorgaben verknüpft: Zum einen die Verpflichtung zur Einbeziehung und zum angemessenen Ausgleich von ökologi-

pellate Body on US-Shrimp, verfügbar unter:

http://www.ciel.org/Publications/shrimpturtlebrief.pdf.

${ }^{65}$ Der Appellate Body zieht hier über die Decision on Trade and Environment die RioErklärung und Bestimmungen der Agenda 21 heran, WTO-Appellate Body, US-Shrimp, WT/DS58/AB/R, 12. Oktober 1998, Rn. 166 ff..

${ }^{66}$ Vgl. im Ansatz auch Centre of Internernational Environmental Law (CIEL), Amicus Brief to the Appellate Body on US-Shrimp, 28, verfügbar unter:

http:/ / www.ciel.org/Publications/shrimpturtlebrief.pdf, die hier zugunsten der USA auf dieses Prinzip verwiesen haben. Die USA hatten die Technologie TED entwickelt, Entwicklungsländer geschult, etc.; vgl. auch Nico Schrijver, The evolution of sustainable development in international law: inception, meaning and status, 2008, 180. 
schen, sozialen und ökonomischen Belangen (festzumachen an entsprechenden internationalen Standards) unter Berücksichtigung der Interessen heutiger und künftiger Generationen (vornehmlich gespiegelt im GabcikovoNagymaros-Fall, Separate Opinion Weeramantry) ${ }^{67}$. Zum anderen die Heranziehung als Auslegungsstandard, der an die normative Matrix des internationalen Nachhaltigkeitskontextes gekoppelt ist und Leitlininen für die Rechtsauslegung an die Hand gibt (vornehmlich gespiegelt im US-Shrimp case). Da sich das Konzept auf das Spannungsverhältnis zwischen ökologischen, sozialen und ökonomischen Parametern bezieht, lassen sich dabei sowohl Argumente für den Investor und dessen Schutzinteressen, als auch für das defensive legitime Regulierungsinteresse des Gastlandes ableiten. Auf Investorseite kann im Kontext des sozialen Faktors etwa das Recht auf Eigentum oder auf einen fairen Prozess ${ }^{68}$ eine Rolle spielen ${ }^{69}$. Ein anderes Beispiel ist das im Nachhaltigkeitskontext fest verankerte Partizipationsprinzip, das Transparenzpflichten und Zugang $\mathrm{zu}$ angemessenen, umfassender und zeitnaher Information verlangt ${ }^{70}$. Dies kann als Verpflichtung des Staates sowohl zugunsten des Investors greifen ${ }^{71}$, als auch ein argumentativer Anknüpfungspunkt sein, wenn es darum geht, für Transparenz und Informationszugang bei Verfahren vor internationalen Schiedsgerichten zu plädieren ${ }^{72}$. Der Gaststaat kann

\begin{abstract}
${ }^{67}$ Auch gefasst als "the principle of integration and interrelationship“, was hier als das Kernanliegen des Nachhaltigkeitskonzepts gefasst wird, in dessen normative Logik sich andere Regierungsstandards einfügen, vgl. zur zentralen Funktion des Integrationsprinzips im Nachhaltigkeitskontext auch International Law Association, Report of the Seventieth Conference, New Delhi (2002), Committee on Legal Aspects of Sustainable Development, 22, 381 (New Delhi Declaration, Prinzip 7). Good Governance und Sustainability bedingen sich gegenseitig, sustainability als Teil von good governance und good governance als Teil von sustainability. Andrew P. Newcombe, Sustainable Development and Investment Treaty Law, in: Journal of world investment \& trade, 2007, 357-407, 398, verfügbar unter: SSRN:

http:/ / ssrn.com/abstract=1016578. Report of the UN-Expert Group on Identification of Principles of International law for Sustainable Development to the CSD, 1995/96, 5, verfügbar unter: http://www.un.org/documents/ecosoc/cn17/1996/background/ecn171996-bp3.htm 1996; Meinhard Schröder, Sustainable Development - Ausgleich zwischen Umwelt und Entwicklung als Gestaltungsaufgabe der Staaten, in: AVR 1996, 251-275, 257; Enquête-

Kommission des Deutschen Bundestages: Konzept Nachhaltigkeit. Vom Leitbild zur Umsetzung, 27 ff.;Marie-Claire Cordonier Segger/Ashfaq Khalfan/Salim Nakjavani,, Weaving the Rules for Our Common Future, Future: Principles, Practices and Prospects for International Sustainable Development Law. Centre for International Sustainable Development Law (CISDL), 2002, 29, verfügbar unter: www.cisdl.org/wtr/pdf/WeavingtheRulesOct2002.pdf.

${ }^{68}$ Menschenrechte kann man zu den sozialen im Zusammenhang mit dem Nachhaltigkeitskonzepts zählen oder auch als Teilaspekt das Konzepts der Guten Regierungsführung, was wiederum ebenfalls zu den gesellschaftsbezogenen Parametern des Nachhaltigkeitskonzepts
\end{abstract} gezählt werden kann, vgl. oben, I, 2.

${ }^{69} \mathrm{Vgl}$. Mondev v. United States, ICSID Case no. ARB/(AF)/99/2, 11 Oktober 2002, Rn. 141 ; Tecnicas Medioambientales Tecmed, S.A. v. United Mexican States, ICSID (Case no.

ARB(AF)/00/2), 29. Mai 2003, Rn. $116 \mathrm{ff.}$.

${ }^{70}$ International Law Association, Report of the Seventieth Conference, New Delhi (2002), Committee on Legal Aspects of Sustainable Development, 22, 381, New Delhi Declaration, Prinzip 5 .

${ }^{71}$ Vgl. Andrew Newcombe, Lluís Paradell, Law and Practice of Investment Treaties: Standards of Treatment, 2009, 284.

72 Luke Eric Peterson, Human Rights and Bilateral Investment Treaties. Mapping the role of human rights law within investor-state arbitration, Rights \& Democracy, 2009, 41, verfügbar 
sich hingegen auf ökologische und soziale Standards (z.B. Menschenrechte) stützen, wenn es darum geht, sein legitimes Regelungsinteresse zu begründen.

Dieses „sowohl als auch“ reflektiert keine „normative Beliebigkeit", wie sie dem Nachhaltigkeitskonzept oft unterstellt wird. Es ist vielmehr Ausdruck des normativen Kerns des Nachhaltigkeitskonzepts ${ }^{73}$, das vor dem Hintergrund des Spannungsverhältnisses zwischen konfligierenden Zielsetzungen mit Blick auf ökonomische (z.B. Investorschutz), soziale und ökologische öffentlichen Anliegen (z.B. in Bezug auf öffentliche Dienstleistungen, Gesundheits- oder Ressourcenschutz) zugunsten oder zulasten verschiedener Beteiligter (Bürger, Investoren) Maßnahmen erfordert, welche den (im Nachhaltigkeitskontext legitimen) Anliegen möglichst weitgehend entgegenkommen (d.h. Synergie stiftend, bzw. trade-off lindernd regulieren). Das Recht kennt Methoden, die rechtliche Beurteilung solcher Art Optimierungsanliegen in Kriterien zu fassen, die es erlauben, das Optimierungsanliegen an handhabbare Beurteilungsparameter zu knüpfen. Ein Beispiel ist der Grundsatz der Verhältnismäßigkeit, wie er im deutschen öffentlichen Recht Anwendung findet. Zwar geht es hier um die "praktische Konkordanz"74 von Grundrechtspositionen in dem Sinne, dass in einer trade-off Situation zwischen zwei Grundrechtspositionen möglichst beide Positionen größtmögliche Berücksichtigung finden sollen; doch ist die dahinter stehende Logik dieselbe ${ }^{75}$. In beiden Fällen geht es um die Pflicht des Entscheidungsträgers, ein möglichst Synergie stiftendes Instrument mit geringem trade-off Potential zu wählen. Aufgrund der Komplexität der Optimierungssituation wird man dabei nie die Maßnahme festlegen können, die „am nachhaltigsten“ oder "am verhältnismäßigsten“ ist, hängt es doch entscheidend von politischen Erwägungen und Überzeugungen ab, welchen Aspekten in der trade-off, bzw. Zielkonfliktsituation mehr Gewicht einzuräumen ist $^{76}$.

Vielmehr geht es darum zu prüfen, ob sich der Entscheidungsträger mit seiner Maßnahme im Rahmen dessen bewegt, was nach Gesichtspunkten der Verhältnismäßigkeit, bzw. im Nachhaltigkeitskontext vertretbar ist. Mit Blick auf das Nachhaltigkeitskonzept ist es etwa bestimmbar und rechtlich überprüfbar, ob der Entscheidungsträger den Kernanforderungen des Nachhaltigkeitskonzepts gerecht wird. Dies heißt zum einen, dass er die in einer Regelungssituation relevanten ökologischen, sozialen und ökonomischen Aspekte sowie langund kurzfristigen Auswirkungen einbezogen und Synergiepotentiale (z.B.

unter: http://www.dd-rd.ca/site/_PDF/publications/globalization/HIRA-volume3ENG.pdf.

${ }^{73}$ Vgl. zu diesem Verständnis Fn. 63.

${ }^{74}$ Begriff geprägt von Konrad Hesse, vgl. ders., Grundzüge des Verfassungsrechts der Bundesrepublik Deutschland, 20. Aufl., Heidelberg 1999, Rn. 72, Peter Lerche, Die Verfassung als Quelle von Optimierungsgeboten?, in: Joachim Burmeister (Hrsg.): Verfassungsstaatlichkeit, In: Burmeister, Joachim (Hrsg.): Verfassungsstaatlichkeit, Festschrift für Klaus Stern zum 65. Geburtstag. München 1997, 197-209, 206.

${ }^{75}$ Vgl. hierzu Katja Gehne, Das Nachhaltigkeitsprinzip als Rechtsprinzip, Fn. 11, Kapitel 5.

${ }^{76}$ Deshalb ist etwa die Frage „Was ist nachhaltig“ ebenso wie „Was ist verhältnismäßig“ konkret-inhaltlich kaum je eindeutig beantworten. 
"milderes Mittel“) und trade-off Aspekte sowie geeignete Maßnahmen berücksichtigt hat. Zum anderen geht es um die normative "Angemessenheit" der Maßnahme, die sich im Rahmen dessen bewegen muss, was nach dem Wertkostüm des Nachhaltigkeitskonzepts gerechtfertigt ist. Die deutsche Dogmatik im Zusammenhang mit dem Verhältnismäßigkeitsgrundsatz unterscheidet hier zwischen der "Tatsachen-“ und der "Wertebene“. Die Tatsachenebene betrifft die technisch-faktischen Fragen: ist eine Maßnahme geeignet, das mit ihr verfolgte Ziel zu erreichen? Gibt es ein Mittel, mit dem das Ziel effizienter oder mit weniger beeinträchtigendem Potential erreicht werden kann (milderes Mittel)? ${ }^{77}$ Die Wertebene betrifft die normativen Fragen: Ist die Maßnahme angemessen in Bezug auf das normative Wertekostüm, auf dem die Rechts- und Gesellschaftsordnung basiert?

Überträgt man diese Dogmatik auf das Spannungsverhältnis zwischen Investitionsschutz und staatlichem Regulierungsinteresse, lässt sich diese Ratio für die rechtliche Argumentation fruchtbar machen. Zunächst muss die Maßnahme in den Anwendungsbereich des Nachhaltigkeitskonzepts fallen, d.h. den Konfliktfall zwischen ökologischer und sozialer Regulierung im öffentlichen Interesse und Investorschutzinteressen (als Ausdruck des makroökonomischen Interesses) betreffen. Aus Sicht des Investors muss eine staatliche Maßnahme im Nachhaltigkeitskontext daher zunächst auf sozialen, ökologischen oder auch ökonomischen Anliegen basieren, die sich im Nachhaltigkeitskontext rechtfertigen lassen. Des Weiteren muss sie die mit dem Investitionsschutz verknüpften normativen Anliegen angemessen berücksichtigen. Dies ist vor dem Hintergrund der staatlichen Pflicht, ökonomische, soziale und ökologische Ziele und Anliegen ${ }^{78}$ so zu berücksichtigen, dass möglichst geringe tradeoff Situationen und möglichst Synergien entstehen, zu beurteilen. In S.D. Myers (ICSID) stellt das Panel z.B. in ähnlicher Weise fest, dass Staaten nach der Rio-Erklärung Handelsbeeinträchtigungen vermeiden sollen, und dass Umweltschutz und ökonomische Entwicklung sich grundsätzlich gegenseitig stützen können und sollen ${ }^{79}$. Wendet man die oben skizzierten Kriterien aus dem dogmatischen Kontext der Verhältnismäßigkeitskontrolle an, muss die Maßnahme geeignet sein, das Regelungsziel zu erreichen (sonst ist sie per se nicht gerechtfertigt), sie muss ein Mittel darstellen, das allen Rechtspositionen möglichst entgegenkommt, d.h. z.B. darf kein Mittel zur Verfügung stehen, dass das Regelungsziel mit angemessenem Aufwand ebenso effektiv erreicht und die Position des Investors erheblich weniger beeinträchtigt ${ }^{80}$, und sie

\footnotetext{
${ }^{77}$ Im deutschen Recht geht es hier um das „mildere Mittel“ mit Blick auf den Grundrechtseingriff, vgl. zu einer Analyse hier Kilian Bizer, Die Ökonomik der Verhältnismäßigkeitsprüfung, 1999, verfügbar unter: http:/ / www.sofiadarmstadt.de/fileadmin/Dokumente/Diskussion/1999/1-99.pdf.

78 Auf der Basis des normativen Wertekostüms des internationalen Nachhaltigkeitskontextes, vgl. hierzu oben unter I. 2.

79 S.D. Myers, Inc. V Government of Canada, First Partial Award on Merits, 13 November 2000, Rn. 247.

${ }^{80}$ Vgl. hierzu z.B. im Rahmen des ",necessity-claims“ Argentiniens im Aguas Argentinas Fall das Argument von Suez, dass Argentinien andere Maßnahmen hätte wählen können, um das Investorinteresse an stabilen Einnahmen und das öffentliche Interesse an niedrigen Wasserpreisen zu erreichen; Suez, Socieded General de Aguas de Barcelona S.A., and Vivendi Uni-
} 
muss (wert-)angemessen sein, was z.B. eher nicht gegeben sein dürfte, wenn ein Investor enteignet wird, weil auf dem Produktionsgelände eine seltene Käferart lebt, die geschützt werden soll ${ }^{81}$. Bei allem ist zu berücksichtigen, dass die juristische Entscheidung angesichts des politischen Gehalts der Fragestellung dem ,judicial self restraint“ insofern unterliegen muss, als der Gaststaat grundsätzlich die Souveränität besitzt, über geeignete Maßnahmen zu entscheiden und die politischen Ziele festzulegen ${ }^{82}$. Sieht man Staaten indes ähnlich wie der IGH ${ }^{83}$ - dem Nachhaltigkeitskonzept verpflichtet, ist er hierbei an ökonomische, soziale und ökologische Nachhaltigkeitskriterien gebunden, die er ",angemessen“, d.h. in gutem Glauben mit Rücksicht auf die jeweiligen im Nachhaltigkeitskontext relevanten Positionen (insofern auch den Investorschutz als ökonomischen Aspekt), zu berücksichtigen hat ${ }^{84}$. Das ,judicial balancing", das bei überlappenden menschen- oder umweltrechtlichen Rechtsbereichen im internationalen Investitionsrecht erforderlich wird, ließe sich auf diese Weise normativ und dogmatisch verankern ${ }^{85}$.

Im Umkehrschluss ergibt sich hieraus, dass Maßnahmen, die sich in dem Angemessenheitsrahmen bewegen und berechtigten sozialen, ökologischen und ökonomischen Zielen im Nachhaltigkeitskontext gelten, grundsätzlich als legitime Regulierungsinteressen betrachtet werden können. Der im Investitionsrecht geläufige Treu und Glauben Aspekt von "legitimate expectations“86, der sich heute vornehmlich auf die Erwartungen des Investors mit Blick auf das Staatsverhalten bei Vertragsabschluss oder Versprechungen und Verhaltensweisen des Staates bezieht, bekäme in diesem Kontext eine andere, normative Konnotation: Legitime Interessen des Investors wären nicht mehr nur im Hinblick auf das Verhalten des Staates zu beurteilen, sondern auch danach, was ein Investor nach den anerkannten Kriterien internationaler Standards mit Blick auf eine gute Regierungsführung, zu der die Beachtung des Nachhaltigkeitskonzepts gehört, von einem Staat erwarten darf. In diese Richtung gehen

versa S.A. v. Argentine Republic, ICSID Case No. Arb/03/19, Reply of Suez, Rn. 508, vgl. Luke Eric Peterson, Human Rights and Bilateral Investment Treaties. Mapping the role of human rights law within investor-state arbitration, Rights \& Democracy, 2009, 29, verfügbar unter: http:// www.dd-rd.ca/site/_PDF/publications/globalization/HIRA-volume3-ENG.pdf..

81 Hier wären aber auch schon sehr wahrscheinlich „mildere“ Maßnahmen, wie z.B. Umsiedlung möglich; das „Tatsachenkriterium“ der zur Verfügung stehenden Mittel und die Wertebene sind hier oftmals überlappend, vgl. Oliver Koch, Der Grundsatz der Verhältnismäßigkeit in der Rechtsprechung des Gerichtshofs der Europäischen Gemeinschaften, 2003, 198 ff..

82 Ian Brownlie, Principles of International law, 2008, 289.

83 Die IGH-Gabcikovo Entscheidung bezieht sich allerdings zunächst nur auf Umweltschutzaspekte, während Judge Weeramantry in seiner Separate Opinion weiter geht, vgl. hierzu oben, II, 2.

84 Vgl. zum Investitionsschutz als Ausdruck des ökonomischen Anliegens im Nachhaltigkeitskontext Andrew P. Newcombe, Sustainable Development and Investment Treaty Law, in: Journal of world investment \& trade, 2007, 357-407, $370 \mathrm{ff} .$.

85 Vgl. hierzu etwa die Beiträge in Pierre-Marie Dupuy, Francesco Francioni, Ernst-Ulrich Petersmann (Hrsg.): Human Rights in International Investment Law and Arbitration, 2009.

86 Vgl. hierzu mwN Andrew Newcombe, Lluís Paradell, Law and Practice of Investment Treaties: Standards of Treatment, 2009, $279 \mathrm{ff}$. . 
z.B. die Fälle S.D. Myers und Methanex ${ }^{87}$, die nahe legen, dass legitime Schutzinteressen bestehen, wenn staatliche Maßnahmen im Sinne des Vorsorgeprinzips ergriffen werden. Auch im Fall SPP v. Egypt kommt ein solches Verständnis zum Ausdruck. Hier geht es um Aktivitäten eines Tourismusunternehmens, die nicht mit der UNESCO Konvention vereinbar sind ${ }^{88}$.

Im Investitionsrecht steht hinter der rechtlichen Beurteilung letztlich vor allem die Frage, wer das Risiko für unvorhergesehene externe Ereignisse, wie z.B. Katastrophen oder Krisen tragen soll, die die Ausgangsbedingungen bei Vertragsschluss verändern, aber auch inwieweit der Staat zu Lasten des Investors und zugunsten öffentlicher Interessen regulieren darf, ohne ausgleichspflichtig zu werden. Letztlich bewegt man sich hier im ur-rechtlichen Raum der Maßstäbe von Treu und Glauben und der hiernach zu beurteilenden Legitimität von Geschäftserwartungen der Investoren. Allgemein besteht die juristische Aufgabe gemäß ihrer Ratio und Verantwortung darin, klare und vorhersehbare Kriterien der rechtlichen Beurteilung zu entwickeln ${ }^{89}$. Das Nachhaltigkeitskonzepts als international gewebtes normatives Wertekostüm, welches das Spannungsverhältnis zwischen ökologischen, ökonomischen und sozialen Gemeinwohlinteressen normativ einhegt, kann hier insbesondere dazu beitragen, eine normative Tiefenstruktur, bzw. eine allgemeine Interpretationsrichtung für die rechtliche Argumentation an die Hand zu geben, die ein gewisses Netz der Vorhersehbarkeit und Kohärenz spannt, wenn es darum geht, die Legitimität von staatlichen Regulierungsinteressen und Investitionsschutzinteressen $\mathrm{zu}$ beurteilen.

Internationale Standards im Nachhaltigkeitskontext können des weiteren de lege ferenda im Hinblick auf die Vertragsgestaltung von IIA ${ }^{90}$ und mit Blick auf die Schiedsgerichtsbarkeit fruchtbar sein, wenn es darum geht, das Spannungsverhältnis zwischen Investorschutz und Interessen staatlicher Regulierung in klarere rechtliche Kriterien zu fassen ${ }^{91}$. Eine wichtige Funktion kann hier etwa die Verankerung des Ziels einer Nachhaltigen Entwicklung in der

87 S.D. Myers, Inc. V Government of Canada, First Partial Award on Merits, 13 November 2000, Rn. 98, Methanex v. US, Final Award, 3 August 2005, Rn. 101 ff., zitiert nach Andrew P. Newcombe, Sustainable Development and Investment Treaty Law, in: Journal of world investment \& trade, 2007, 357-407, $378 \mathrm{ff}$. .

88 Southern Pacific Properties Limited v. Egypt, ICSID Case, ARB/84/3, 20. Mai 1992, Rn. 154.

${ }^{89} \mathrm{Vgl}$. H. L. A. Hart: The concept of law, 2. Aufl. Oxford 1994, $240 \mathrm{ff} .$.

90 Vgl. für einen Überblick, Aaron Cosbey, Howard Mann, Luke Eric Peterson, Konrad v. Moltke, Investment and Sustainable Development: A Guide to the Use and Potential of International Investment Agreements, International Institute for Sustainable Development (IISD), 2004; vgl. auch den vorgeschlagenen Modell-Vertrag http://www.iisd.org/pdf/2005/investment_model_int_agreement.pdf.

${ }_{91} \mathrm{Vgl}$. Andrew P. Newcombe, Sustainable Development and Investment Treaty Law, in: Journal of world investment \& trade, 2007, 357-407; Nathalie Bernasconi, Background paper on Vattenfall v. Germany arbitration, IISD 2009, verfügbar unter:

http://www.iisd.org/pdf/2009/background_vattenfall_vs_germany.pdf ; Konrad v. Moltke, An International Investment Regime? Issues of Sustainability, IISD 2000; Markus W. Gehring, Sustainable International Trade, Investment and Competition Law, in: Marie-Claire Cordonier Segger,Ashfaq Khalfan (Hrsg.): Sustainable Development Law, Principles, Practices, \& Prospects, 2004, 281-294. 
Präambel oder den Zielbestimmungen des Vertrages übernehmen. Über Art. 31 Abs. 1 WVK folgt daraus, dass der Vertrag im Licht des Nachhaltigkeitskonzepts auszulegen ist. Über eine Verankerung des Nachhaltigkeitskonzepts in IIA, insbesondere im Kontext regionaler und multilateraler Kooperation oder auch auf der Ebene der Schiedsgerichtsbarkeit (anwendbares Recht, Auslegungsprinzipien), könnte über das mit dem Nachhaltigkeitskonzept verknüpften Wertekostüm ein Standard-Mainstreaming erreicht werden, das ein erhebliches Potential der Kohärenzwirkung birgt. Dies in Kombination mit sorgfältig erarbeiteten, konkretisierenden Ausnahmeklauseln und Auslegungsleitlinien (good faith standards) könnte $\mathrm{zu}$ mehr Klarheit und Vorhersehbarkeit beitragen, wenn es um die Beurteilung legitimer Regulierung geht. Über die Einfügung von Ausnahmeklauseln entlang von Standards aus dem sozialen und ökologischen Gemeinwohlkontext wäre insbesondere Klarheit darüber geschaffen, unter welchen Umständen die Ausgleichspflichtigkeit des Gaststaates nicht gegeben ist. In diesem Kontext ließe sich auch mit einer Gemeinwohlverantwortung des Investors arbeiten, welche die Legitimität staatlicher Regulierungsinteressen im Nachhaltigkeitskontext begründet ${ }^{92}$. Einen Beitrag könnte auch die Verankerung prozeduraler Pflichten in IIAs leisten, welche die Durchführung von Nachhaltigkeitsprüfungen im Vorfeld von Investitionsmaßnahmen verlangen ${ }^{93}$. Diese könnten zum einen für mehr Transparenz bezüglich der Beurteilung legitimer Erwartungen sorgen, zum anderen erlaubten sie mehr Raum für informierte politische Entscheidungen im Vorfeld von Investitionsmaßnahmen, so dass eine Regelung darüber getroffen werden kann. Derartige Analysen sind z.B. bereits verpflichtend für Investitionsgarantien im Zusammenhang der International Finance Cooperation (IFC) ${ }^{94}$. Ein anderer Aspekt sind Nachhaltigkeitsprüfungen im Hinblick auf die Auswirkungen von Bestimmungen internationaler Investitionsabkommen. Im EU-Kontext unterliegen etwa bereits alle EU Politiken einer Nachhaltigkeitsprüfung ${ }^{95}$, weshalb z.B. bereits internationale Handelsabkommen auf ihre

\footnotetext{
92 Vgl. - allerdings bisher lediglich bezogen auf den menschenrechtlichen Kontext - die

"responsibility to respect" von Unternehmen, die mit Unternehmenserwartungen entgegen "good sustainable governance" kaum vereinbar wäre. Im Juni 2008 hat der UN Menschenrechtsrat eine Resolution verabschiedet, wonach "transnational corporations and other business enterprises have a responsibility to respect human rights", vgl. Human Rights Council Resolution 8/7, 18 June 2008; vgl. John Ruggie 'Protect, Respect and Remedy: A Framework for Business and Human Rights', in: Innovations: Technology, Governance, Globalization (2008), 189-212; interessant ist hier eine Parallele zu der "Sozialpflichtigkeit des Eigentums" im deutschen Recht, vgl. hierzu Nathalie Bernasconi, Background paper on Vattenfall v. Germany arbitration, IISD 2009, verfügbar unter:

http://www.iisd.org/pdf/2009/background_vattenfall_vs_germany.pdf, S. 4; Walter Leisner, Sozialbindung des Eigentums, 1972.

${ }^{93}$ Andrew P. Newcombe, Sustainable Development and Investment Treaty Law, in: Journal of world investment \& trade, 2007, 357-407, verfügbar unter SSRN: http:/ / ssrn.com/abstract $=1016578$.

${ }^{94}$ Vgl. http://www.ifc.org/ifcext/sustainability.nsf/Content/EnvSocStandards.

${ }^{95}$ Communication from the Commission on Impact Assessment, Brussels, 5.6.2002 COM(2002) 276 final.
} 
Nachhaltigkeitsimplikationen überprüft und flankierende Maßnahmen für trade-off Situationen vorgeschlagen werden ${ }^{96}$.

\section{Fazit}

Mit Blick auf die Vertragsbestimmungen von Lissabon, Artikel 205-207 in Verbindung mit 21 Abs. 2 (d) AEUV, rückt das Nachhaltigkeitskonzept als Leitbild der europäischen Außenpolitik im entwicklungspolitischen Kontext auf die Agenda. Dies kann nicht nur Konsequenzen für die Rechtsauslegung haben, sondern auch Anlass dafür sein, die künftigen IIAs der EU und ihrer Mitgliedstaaten im Lichte des Nachhaltigkeitskonzepts auszugestalten und anzuwenden. Wie gesehen, birgt das Nachhaltigkeitskonzept Kohärenz stiftende Leitlinien, wenn es darum geht, das Spannungsverhältnis zwischen staatlicher Regulierung im Gemeinwohlinteresse und Investitionsschutzinteressen mit Kriterien anzureichern, die dazu beitragen können, die Legitimität der Interessen vor dem Hintergrund widerstreitender Rechtsordnungen und Paradigmen in (relativ vorhersehbare) rechtliche Kategorien zu fassen. Nimmt die EU ihre Nachhaltigkeitszielsetzung und - Politik ernst, kann sie hier in einer Vorreiterrolle einen wichtigen Beitrag zu einer kohärenten, im Einklang mit internationalen Standards stehenden Rechtsentwicklung leisten.

${ }^{6}$ Siehe http://ec.europa.eu/trade/wider-agenda/development/sustainability-impactassessments/. 